\title{
Theory and simulation of the swelling of polymer gels
}

\author{
N. R. Kenkare, ${ }^{\text {a) }}$ C. K. Hall, ${ }^{\text {b) }}$ and S. A. Khan \\ Department of Chemical Engineering, Box 7905, North Carolina State University, \\ Raleigh, North Carolina 27695-7905
}

(Received 18 October 1999; accepted 4 April 2000)

\begin{abstract}
A combined discontinuous molecular dynamics and Monte Carlo simulation technique is used to study the swelling of athermal, continuous-space, near-perfect, trifunctional polymer networks containing hard chains of lengths 20 and 35 immersed in an athermal hard-sphere solvent. The swelling simulations are conducted under conditions of constant pressure and chemical potential. A simple, analytical theory for gel swelling is presented in which the gel free energy is calculated as the sum of an elastic, affine-type term, and a liquidlike mixing term that is based on the generalized-Flory dimer equation of state. The theory shows good agreement with our simulation results for the gel properties at swelling equilibrium. (c) 2000 American Institute of Physics.

[S0021-9606(00)50425-5]
\end{abstract}

\section{INTRODUCTION}

When placed in a low molecular weight solvent, all crosslinked polymers will absorb the solvent and swell. The swelling behavior of crosslinked polymers has led to their widespread application in drug delivery, molecular separation systems, biomedical devices including implants and contact lenses, and in emerging technologies such as gelbased valves and actuators, sensors, artificial muscles and display devices. ${ }^{1}$

The aim of this paper is to investigate the molecular mechanisms underlying gel swelling using carefully controlled computer simulations. Specifically, we use a combination of molecular dynamics and Monte Carlo simulation techniques to investigate the swelling of athermal gels in athermal, monomeric solvents. We also derive an analytical, molecularly based theory for gel swelling by extending our previous work ${ }^{2}$ on the pressure-volume properties of solventfree polymer networks to the case in which solvent is present.

One of the first molecularly based approaches to gel swelling was that of Flory and Rehner, ${ }^{3}$ who suggested that the change in Helmholtz free energy of a polymer gel upon swelling could be expressed as the sum of a polymer-solvent mixing free energy term, and an elastic free energy term,

$$
\Delta A_{\text {swell }}=\Delta A_{\text {mixing }}+\Delta A_{\text {elastic }},
$$

where $\Delta A_{\text {mixing }}$ is the free energy change on mixing of the equivalent uncrosslinked polymer and solvent, and $\Delta A_{\text {elastic }}$ is the elastic free energy change due to the configurational rearranging and stretching of the crosslinked network chains during the swelling process. The elastic free energy term is considered to be completely independent of the presence and nature of the solvent except insofar as the absorption of solvent by the gel causes the network to expand and its chains to stretch. In the Flory-Rehner approach, the polymer-

\footnotetext{
${ }^{a}$ Currently at Lucent Technologies, Norcross, GA.

b) Author to whom correspondence should be addressed.
}

solvent mixing free energy term is obtained from the FloryHuggins theory, ${ }^{4}$ and the elastic free energy term is obtained from the affine model for rubber elasticity. ${ }^{5,6}$ Most subsequent theoretical work on gel swelling has been based on the Flory-Rehner development [i.e., on Eq. (1)], although different forms of the mixing and elastic contributions have been used. ${ }^{7-10}$

Much of the theoretical work on the swelling of crosslinked systems applies to perfect networks, i.e., networks with constant chain length between junctions and few structural irregularities (dangling chains, loops, or junctions of varying functionality). The validation of network theories is best done using model near-perfect networks with wellcharacterized molecular structure. Experimental efforts in this direction are, however, hampered by the difficulty associated with obtaining precise measurements of the network structure. Over the past decade, computer simulation has proved to be a uniquely useful tool in investigating the properties of polymer networks since it allows the construction and investigation of near-perfect model networks with wellcharacterized structure.

Simulation studies of polymer networks have largely focused on network dynamics, crosslinking kinetics and on PVT properties. ${ }^{11-18,2,19-21}$ Simulations of gel swelling are rare, partly because of the relatively complex simulation techniques required, and partly because gels take very long times to reach swelling equilibrium. As a result, swelling simulations require the use of efficient simulation techniques as well as large amounts of computing time.

To our knowledge, the only simulations of gel swelling are those of Escobedo and de Pablo, ${ }^{21,22}$ who conducted Monte Carlo simulations of the swelling of perfect, tetrafunctional, athermal networks (of chain lengths 10 to 32) having a diamondlike junction connectivity immersed in athermal monomeric and short-chain solvents. The thermodynamic properties of the solvent phase were obtained using a suitable equation of state (for instance the Carnahan-Starling ${ }^{23}$ equation of state for monomeric solvents) and the thermodynamic properties of the gel phase 
were obtained by conducting Monte Carlo simulations at constant external chemical potential and pressure, i.e., the $\mu \mathrm{PT}$ ensemble. Owing to the limitations of conventional Monte Carlo methods for simulating crosslinked systems, Escobedo and de Pablo invented a set of complex Monte Carlo moves (the ECCB, slab, and cluster moves) for relaxing the network and for conducting the volume change moves necessary in the isobaric ensemble. They found that the mole fraction of the solvent in the gel increased with pressure and with network chain length, and that the gel packing fraction increased with pressure and decreased with network chain length. By conducting a set of NPT ensemble simulations of the gels as they approached equilibrium at a particular pressure, Escobedo and de Pablo found that the packing fraction of the gel always lies between that of the dry network and the bulk solvent at the same pressure. They also presented a mean-field theory for gel swelling, in which the free energy of the gel was considered to be the sum of the free energy of a reference polymer/solvent mixture, and the elastic free energy of the solvent-free network. The free energy of the reference polymer/solvent mixture was obtained from an equation of state for athermal molecules introduced in a previous publication by the authors, ${ }^{24}$ and the elastic free energy of the network was evaluated directly from their simulation results. The Escobedo and de Pablo theory fits their simulation results fairly well; however its semicomputational nature precludes its general use for predicting swelling equilibria. Their work in simulating gel swelling is significant in that it presents a way to overcome the limitations of conventional Monte Carlo techniques in investigating these systems. Escobedo and de Pablo's work represents the first successful simulation-based investigation of gel swelling.

Our study of gels is motivated by the paucity of simulation data on the swelling and structural properties of network systems, and by the need for an improved analytical theory to predict the phase equilibrium properties of polymer gels. The purpose of this paper is to describe our novel hybrid Monte Carlo molecular dynamics technique for simulating the swelling of model gels, to present data for the solvent uptake and change in volume of model trifunctional gels upon swelling, and to propose a simple theoretical model for gel swelling based on our previously derived equation of state for solvent-free networks. ${ }^{2}$

We use discontinuous molecular dynamics techniques to construct near-perfect, off-lattice, tri-functional networks of chain lengths 20 and 35 . The swelling of these networks in athermal solvents is simulated by allowing the network to absorb hard-sphere solvent molecules and to expand under conditions of constant external chemical potential and pressure, where the chosen external chemical potential and pressure correspond to the chemical potential and pressure of the pure (bulk) solvent. This procedure is akin to placing the gel in a large excess of solvent and letting it swell, and is similar to the procedure used by Escobedo and de Pablo ${ }^{21,22}$ in their gel swelling simulations. In this work, the swelling of the gel is simulated using a combination of the discontinuous molecular dynamics (DMD) method and the $\mu \mathrm{PT}$ ensemble Monte Carlo algorithm. The DMD method is used to dis- place the solvent and gel segments in order to relax the system, and the $\mu \mathrm{PT}$ Monte Carlo algorithm is used to conduct volume change and particle insertion/deletion moves which are necessary for the gel to achieve mechanical and chemical equilibrium with the bulk solvent. The thermodynamic properties of the bulk solvent are assumed to remain constant throughout the swelling process, and are described by the Carnahan-Starling ${ }^{23}$ equation of state for hard spheres. Our combined simulation technique is fairly efficient and allowed us to simulate gel systems containing more than 12000 segments. Another advantage of using this technique is that it can be used to simulate the swelling of networks of different functionalities and molecular weights, without the introduction of complex, system-specific moves.

Highlights of our simulation results are as follows. We find that the solvent site fraction (defined as the ratio of the number of solvent beads to the total number of segments in the gel) and the gel packing fraction increase with pressure and chemical potential. The solvent site fraction also increases with chain length while the gel packing fraction decreases with chain length. During the constant-pressure swelling of the gel to its equilibrium state, the gel packing fraction is always found to lie between that of the dry network and of the bulk solvent. These observations are all in qualitative agreement with the results from the simulations of Escobedo and de Pablo's. Quantitatively, our results differ somewhat from those of Escobedo and de Pablo; for instance, the solvent site fraction in our swelled gels shows a weaker dependence on chain length than in their systems. We also examine the structural characteristics of the network chains as the gel swells, and find that the mean-squared endto-end vectors of the chains exhibit a weaker-than-affine scaling behavior with the macroscopic swelling.

We extend our previously published theory of networks ${ }^{2}$ to the case of gel swelling. As before, the network is modeled as a set of interpenetrating dendrimers, where each dendrimer branch is a network chain. The free energy of the gel is taken to be the sum of a liquidlike contribution which arises from the polymer-solvent mixing interaction and an elastic contribution which arises from the change in configurational free energy of the chains during the volume change that accompanies swelling. The liquidlike contribution to the free energy of the dendrimer-solvent system is obtained from the Generalized Flory-Dimer equation of state for mixtures. ${ }^{25}$ The elastic free energy contribution to the free energy of the system is obtained the same way as in our previous paper. In this method we invoke an analogy between a classical spring and an ideal, Gaussian network chain, obtain an equivalent "spring constant" and hence free energy for dendrimers composed of these chains, and calculate the network elastic free energy (for the deformed and undeformed network) as the sum of the free energies of the constituent dendrimers.

We use our theory to predict the gel packing fraction and solvent site fraction at swelling equilibrium over a range of external solvent pressures. The theory shows very good agreement with our simulation data except at high pressures. It is also remarkably successful in predicting the amount of solvent taken up by the gel and the change in the gel volume 
as the system moves along the constant-pressure path to swelling equilibria.

The organization of our paper is as follows. Section II describes the molecular model and the simulation techniques used for network construction and relaxation, and for gel swelling. In Sec. III, our theoretical model is described, and in Sec. IV, our simulation and theoretical results are discussed. A brief summary and some further discussion are given in Sec. V.

\section{MOLECULAR MODEL AND SIMULATION TECHNIQUE}

In this paper, we combine the discontinuous molecular dynamics (DMD) technique that we have used earlier to treat polymer melts ${ }^{26,27}$ and networks, ${ }^{18,2}$ with the $\mu \mathrm{PT}$ ensemble Monte Carlo algorithm. We begin this section by describing the molecular model for the network and solvent, and then review the DMD technique as it is applied to polymer melts. Next we outline our method for the construction, relaxation, and evaluation of the pressure-volume properties of solventfree networks. We conclude this section by describing the technique that we have used to simulate gel swelling.

The basic unit of the polymer network is a polymer chain which we represent as a freely jointed chain of tangent hard spheres. The potential energy of interaction between any two beads (segments) on the same or neighboring chains in the network is given by

$$
\begin{array}{rlrl}
U(r) & =0, & r>\sigma, \\
& =\infty, \quad r \leqslant \sigma,
\end{array}
$$

where $\sigma$ is the bead diameter. The solvent molecules are represented as hard spheres having the same diameter as the network beads. The potential energy of interaction between any two solvent beads, or between a solvent bead and a bead on a network chain is given by Eq. (2).

\section{A. DMD technique, network construction and $P-V$ properties}

It is difficult to simulate a system of tangent hard sphere chains using standard molecular dynamics techniques because the tangency requirement cannot be implemented directly. To circumvent this problem, Rapaport ${ }^{28}$ and Bellemans ${ }^{29}$ suggested an approach in which the bond length, $l$, between successive segments of the tangent hardsphere chain is not kept constant, but is instead allowed to vary freely over the range, $\sigma(1-\delta)<l<\sigma(1+\delta)$ where $\delta \ll 1$. The chain then effectively becomes a collection of segments connected by sliding links (the Bellemans chain). As $\delta \rightarrow 0$, the chain approximates the tangent-hard-sphere chain model. The relaxation of the constant bond length requirement allows the chain segment trajectories to be partially decoupled, resulting in linear trajectories between collisions. The system dynamics can then be treated using the discontinuous molecular dynamics (DMD) techniques developed by Alder and Wainwright ${ }^{30-32}$ for hard sphere systems. A comprehensive description of the discontinuous molecular dynamics technique is given in the paper by Smith et al., ${ }^{26,27}$
TABLE I. The network structural characteristics in terms of the chain length, $n$, number of chains, $N_{c}$, the total number of segments in the system, $N_{t}$, the elastic fraction, $\%_{\mathrm{el}}$, the number of trifunctional junctions, $N_{3}$, the number of unsaturated (bifunctional) junctions, $N_{2}$, the number of dangling ends, $N_{\text {end }}$, the number of loops, $N_{\text {loop, }}$ and the number of free chains, $N_{\text {free }}$.

\begin{tabular}{ccccccccc}
\hline \hline$n$ & $N_{c}$ & $N_{t}$ & $\%_{\text {el }}$ & $N_{3}$ & $N_{2}$ & $N_{\text {end }}$ & $N_{\text {loop }}$ & $N_{\text {free }}$ \\
\hline 20 & 90 & 1800 & 0.99 & 60 & 0 & 0 & 1 & 0 \\
35 & 90 & 3150 & 0.96 & 59 & 1 & 2 & 1 & 0 \\
\hline \hline
\end{tabular}

along with descriptions of various methods for improving the code efficiency, including neighbor lists, linked lists, binary trees, and delayed position updates.

In order to construct solvent-free tri- or tetrafunctional networks we endlink melts of linear Bellemans chains. Since it is easier to achieve near-complete extents of reaction at lower densities than at higher densities, the endlinking is conducted at an initial system packing fraction of $\eta=0.3$. The packing fraction is then increased to $\eta=0.45$ by growing the segment diameter. The system packing fraction is defined to be

$$
\eta=\frac{\pi}{6} \frac{N_{c} n \sigma^{3}}{V},
$$

where $N_{c}$ is the number of network chains, $n$ is the chain length and $V$ is the volume of the simulation cell. This crosslinking method allows us to construct nearly perfect dry networks, i.e., solvent-free systems containing a very small percentage of free chains, dangling ends, loops and unsaturated junctions (junctions to which only two chain ends are attached). Details of the crosslinking procedure are described in a previous publication. ${ }^{18}$

In this paper, we focus on two such trifunctional networks, one containing chains of length 20 and the other containing chains of length 35. Each network contains 90 chains. The chain segment diameter equals 1, the Bellemans bond variation factor, $\delta$ equals 0.05 , and the box length varies with the packing fraction. The structural characteristics of the networks, displayed in Table I, are described in terms of the elastically active fraction, $\%_{\text {elastic }}$, the number of trifunctional junctions, $N_{3}$, the number of bifunctional (unsaturated) junctions, $N_{2}$, the number of dangling ends and loops, $N_{\text {end }}$ and $N_{\text {loop }}$ and the number of free chains, $N_{\text {free }}$. The elastically active fraction is an important measure of the "perfectness" of our networks, and is defined to be the fraction of the chains that are connected at both ends to junctions with at least three independent paths to the network. ${ }^{33,34}$ It is calculated in the table by subtracting the number of unsaturated junctions, dangling chains, and loops from the total number of network chains, and dividing by the total number of network chains. Since the elastically active fraction is close to 1 for both of the networks considered, and the number of dangling chains, loops and unsaturated junctions is low, we consider these networks to be near-perfect systems.

Once the networks attained their final packing fraction of 0.45 , they were relaxed for 500 million collisions. Their compressibility factors were then evaluated from the Clausius virial theorem in the following form: 
TABLE II. Simulation results (Ref. 2) for the reduced pressure, $P^{*}$ of dry trifunctional networks of chain length 20 and 35 .

\begin{tabular}{ccc}
\hline \hline$\eta$ & $P^{*}(n=20)$ & $P^{*}(n=35)$ \\
\hline 0.45 & 5.129 & 5.149 \\
0.40 & 3.021 & 3.042 \\
0.35 & 1.736 & 1.755 \\
0.30 & 0.959 & 0.973 \\
0.25 & 0.495 & 0.507 \\
0.15 & 0.087 & 0.095 \\
0.05 & -0.0065 & -0.00098 \\
0.03 & -0.0107 & -0.0045 \\
0.01 & -0.0224 & -0.0074 \\
0.005 & & -0.012 \\
\hline \hline
\end{tabular}

$$
Z^{\mathrm{net}} \equiv \frac{P V}{N^{\mathrm{net}} k_{B} T}=N_{c} n-\frac{m \Sigma_{\mathrm{coll}} \mathbf{r}_{i j} \cdot \Delta \mathbf{v}_{i j}}{3 k_{B} T t_{e}},
$$

where $P$ is the network pressure, $N^{\text {net }}$ is the number of network molecules $\left(N^{\text {net }}=1\right.$ since the network is considered to be one large molecule), $t_{e}$ is the elapsed simulation time over which the sum is calculated, $k_{B}$ is the Boltzmann constant, $T$ is the temperature, $m$ is the mass of a network chain segment, $\mathbf{r}_{i j}$ is the vector between segment centers at a collision, and $\Delta \mathbf{v}_{i j}$ is the velocity change for the colliding pair. In this paper, the simulation results are reported in terms of the reduced pressure, $P^{*} \equiv P \sigma^{3} / k_{B} T=Z^{\text {net }} N^{\text {net }} 6 \eta /\left(\pi N_{c} n\right)$.

The evaluation of the pressure-volume properties of solvent-free networks is a useful intermediate step in the study of the swelling properties of solvent-containing networks (gels). The $P-V$ properties of solvent-free trifunctional networks of chain lengths, $n=20,35,50$, and 100, were reported by us in a previous publication. ${ }^{2}$ The network pressure was obtained over a range of packing fractions (i.e., volumes) by starting with a high initial density network and expanding it to lower densities using a volume change algorithm that takes advantage of the variable bond-length property of the Bellemans chain. Details of this algorithm are described in Ref. 2. Employing this volume-change method, the networks were expanded from their initial packing fraction of 0.45 to successively lower packing fractions of $0.35, \ldots, 0.003$, and the network pressures were calculated at each packing fraction. These simulation results for the network pressure are reproduced in Table II and will be used in a comparison later in this paper (cf. Sec. IV) with the pressure-volume behavior of gels.

\section{B. Gel swelling}

In this section we describe our combined $\mathrm{DMD} / \mu \mathrm{PT}$ ensemble Monte Carlo algorithm for simulating the swelling of athermal networks in hard-sphere solvents.

The application of modern phase equilibria computer simulation techniques (e.g., the Gibbs ensemble method) to the case of gel swelling typically involves the use of two separate simulation cells, one for the bulk solvent phase and one for the gel phase. This can be reduced to the use of a single simulation cell for the gel phase alone by considering the situation in which the swelling of the gel takes place in a large excess of bulk solvent. Since the amount of bulk sol- vent is large compared to the amount of solvent taken up by the gel during swelling, the thermodynamic properties of the bulk solvent (pressure, chemical potential, and temperature) can be assumed to remain constant throughout the swelling process. Therefore the bulk solvent phase need not be simulated since its thermodynamic properties can be obtained by using an accurate equation of state. In this paper we use the Carnahan-Starling ${ }^{23}$ equation of state for hard sphere solvents. The simulation of gel swelling in the bulk solvent then reduces to the simulation of the gel phase alone under conditions of constant external (bulk solvent) chemical potential, pressure, and temperature, i.e., in the $\mu \mathrm{PT}$ ensemble. At the start of the simulation the gel phase may contain some or no solvent. To achieve thermal, mechanical and chemical equilibrium between the gel and bulk solvent phases in the $\mu \mathrm{PT}$ ensemble, the gel undergoes a number of particle displacement, volume-change, and solvent particle insertion/deletion moves. After a sufficient number of such moves, the gel approaches swelling equilibrium, i.e., the pressure, and the solvent chemical potential of the gel converge to stable values that are equal to the bulk solvent pressure and chemical potential (the thermal equilibrium condition is trivially satisfied in an athermal system).

In this paper, we have combined the DMD technique with the $\mu$ PT ensemble Monte Carlo algorithm in such a way that the DMD technique is used within the framework of the $\mu \mathrm{PT}$ algorithm to execute the particle displacement and volume change moves, while purely Monte Carlo algorithm steps are used for the particle insertion/deletion moves. Our method departs from standard phase equilibria techniques in which Monte Carlo algorithm steps are used for all three types of moves. Our motivation for developing this hybrid method is the following: since the structure of the network is highly constrained and complex, conventional Monte Carlo particle displacement and volume change moves cannot be used because they would cause bond breakage and destruction of the network topology. Thus there is a need for developing alternative techniques/moves for network systems. One solution to this problem is to develop a set of complex, system-specific Monte Carlo particle displacement and volume-change moves that would preserve the network topology, as was done by Escobedo and de Pablo. ${ }^{21,22}$ Our solution has been to simply use the DMD method (which we have employed earlier to investigate the dynamics and pressure-volume properties of networks ${ }^{18,2}$ ) to conduct the displacement and volume-change moves within the framework of the $\mu \mathrm{PT}$ Monte Carlo algorithm. The idea of combining the DMD method with MC techniques is not novelfor instance a combined DMD-NPT ensemble method was used by Wilson and Allen ${ }^{35}$ to investigate liquid crystal formation. This is, however, the first time that such an approach has been used to study gel swelling. The advantage of our method is that is simple and yet general, so that it can be used to investigate phase equilibria in a variety of constrained systems.

The details of our simulation technique are as follows. In order to use the DMD technique within the constant $\mu \mathrm{PT}$ ensemble MC algorithm, we must assign each particle a velocity and calculate particle collision times, just as in a 
purely DMD simulation. At first glance, the simplest way to combine the DMD and $\mu \mathrm{PT}$ techniques would perhaps be to replace the particle displacement moves in a regular NVT ensemble simulation with DMD events (particle pair collisions or bond stretches). The drawback to this simple procedure is that the time interval between events in a DMD simulation is not equal (since the simulation proceeds on an event-by-event basis), which in the combined $\mu$ PT-DMD algorithm has the effect of introducing a bias which disturbs the approach of the system to equilibrium. In our simulations, we have avoided this bias in the following manner. The total simulation time is divided into equal time steps, which are then grouped into cycles of $N_{\text {step }}$ time steps each. The system is allowed to begin the DMD simulation (i.e., the particle displacement steps). When the simulation time is equal to the duration of a time step (or a multiple of a time step), a Monte Carlo move is attempted. The Monte Carlo move may be either a particle displacement (attempted as a DMD event), a volume change or a particle insertion/ deletion. The move is accepted or rejected in accordance with the criteria described in the following paragraphs. After the conclusion of the move, the time lists (and particle positions and velocities if necessary) are recalculated and the MD simulation is resumed, until the simulation time is equal to that of the next time step, when the next MC move is attempted. Thus in this procedure, a small number of additional DMD particle displacement events are conducted between successive MC moves. The actual number of DMD events taking place between MC moves depends on the size of the time step, i.e., if the time step is chosen to be sufficiently small, very few or no DMD events will occur between MC moves. However, the time step must be chosen to be large enough that the system approaches equilibrium in a finite number of moves. In our simulations, between 0 and 4 DMD events occurred between MC moves. The specific type of MC move that is attempted (i.e., particle displacement, volume change or particle insertion/deletion moves) is selected randomly, but in accordance with a preset probability that ensures that each cycle of $N_{\text {step }}$ Monte Carlo moves consists of $N_{\text {disp }}$ particle displacement moves, $N_{\text {vol }}$ volume change moves, and $N_{\text {ins }}$ particle insertion/deletion moves.

Starting with a dry network or with a gel containing some solvent, several million Monte Carlo moves are carried out during the slow approach of the system to equilibrium. The simulation is continued until the pressure of the gel and the solvent chemical potential converge to stable values that are approximately equal to the corresponding bulk solvent values. After the pressure and solvent chemical potential of the gel have remained stable about the corresponding bulk solvent values for several million Monte Carlo moves, the system is considered to have reached equilibrium. The equilibrium state of the gel is described by its pressure, solvent chemical potential, gel packing fraction (which indicates the magnitude of the volume change due to swelling), and solvent site fraction, which is defined as the ratio of solvent segments to the total number of segments (network plus solvent) in the gel, and is a measure of the amount of solvent absorbed by the gel.

The details of the three types of simulation moves used, i.e., the particle displacement, volume change and particle insertion/deletion moves are given below. During the particle displacement moves, the DMD procedure is utilized to displace the network and solvent beads, keeping the composition and volume of the simulation cell constant. During the volume change move, a trial volume change is attempted, keeping the composition of the cell unchanged. A random number, $\epsilon$, is chosen from the range $\left(-\epsilon_{\max }, \epsilon_{\max }\right)$, and the new box volume, $V_{\text {new }}$, is taken to be

$$
V_{\text {new }}=V_{\text {old }}(1+\epsilon)^{3} \text {, }
$$

where $V_{\text {old }}$ is the old box volume. The positions of the constituent molecules must be scaled in accordance with the change in volume of the cell. In conventional constantpressure simulations of uncrosslinked chainlike systems, the positions of the particles in the box during a volume change move can be changed by scaling the center of mass locations of each chain molecule by $(1+\epsilon)$, and moving the whole chain without altering the intramolecular positions of the segments. For crosslinked systems, this method cannot be used since it would cause the network connectivity to be broken. To get around this problem, we take advantage of the flexibility of the Bellemans bond length and simply scale the position of each segment by the factor $(1+\epsilon)$. The volume change move is rejected if it results in bond breaking or overlaps between the segments. The move is accepted with a probability equal to $\min \left[1, \exp \left(-\Delta H / k_{B} T\right)\right]$, where $\Delta H$ is given by

$$
\Delta H=\Delta U+P \Delta V-N_{t} k_{B} T \log \left(\frac{V_{\text {new }}}{V_{\text {old }}}\right),
$$

where $\Delta U$ is the change in internal energy due to the volume change (equal to zero or infinity for a hard-sphere system), $\Delta V=V_{\text {new }}-V_{\text {old }}$ is the change in volume of the system, and $N_{t}$ is the total number of segments in the system. Thus, for a volume change move to be accepted, $\Delta U$ must be zero, i.e., after an attempted volume change, we must search through all the particles in the system to ensure that there are no overlaps or broken bonds. The quantity $\epsilon_{\max }$ is chosen so as to produce an acceptance ratio ${ }^{36}$ of $35-50 \%$. Once the volume change move is accepted, all the collision times in the system are recalculated and the neighbor lists are reset.

During the particle insertion/deletion move, attempts are made to insert or delete a solvent molecule from the system (with equal probability), keeping the volume of the simulation cell constant. During a particle insertion move, a trial particle is created at a random position in the simulation cell, and the move is accepted ${ }^{36}$ with a probability equal to $\min \left[1, \exp \left(-\Delta C / k_{B} T\right)\right]$ where

$$
\Delta C=\Delta U-\mu-\log \left(\frac{V}{N_{s}+1}\right),
$$

where $\mu$ is the chemical potential of the bulk solvent and $N_{s}$ is the number of solvent molecules. Just as in the volume change move, the acceptance of a particle insertion move involves searching through all of the particles in the system to ensure that there are no overlaps. Once the move is accepted, the new particle is assigned a random velocity from a Gaussian distribution, and all the particle velocities are res- 
caled to ensure zero total momentum. During a particle deletion move, a randomly chosen particle is destroyed; this move is accepted with a probability equal to $\min [1$, $\left.\exp \left(-\Delta D / k_{B} T\right)\right]$ where

$$
\Delta D=\Delta U+\mu+\log \left(\frac{V}{N_{s}}\right)
$$

The particle velocities are then rescaled as before.

The gel pressure and solvent chemical potential are evaluated at regular intervals during the simulation in order to monitor the approach of the system towards equilibrium. The gel pressure is obtained by evaluating the compressibility factor from the Clausius virial theorem in the following form:

$$
Z=\frac{N_{t}}{N_{m}}-\frac{m \Sigma_{\mathrm{coll}} \mathbf{r}_{i j} \cdot \Delta \mathbf{v}_{i j}}{3 N_{m} k_{B} T t_{e}},
$$

where $N_{t}=N_{c} n+N_{s}$ is the total number of beads (network segments and solvent), $N_{s}$ is the number of solvent beads, and $N_{m}=1+N_{s}$ is the total number of molecules in the system. The reduced pressure of the system, $P^{*}$, is obtained from the relation, $P^{*}=P \sigma^{3} / k_{B} T=Z 6 \eta N_{m} / \pi N_{t}$. The excess chemical potential of the solvent in the gel is calculated using the Widom test-particle insertion method ${ }^{37}$ in which we repeatedly attempt to insert a test solvent particle at random positions in the gel. For a hard-sphere system, the excess chemical potential of the solvent in the gel is simply equal to ${ }^{37} k_{B} T \ln \left(p_{s}^{\text {ins }}\right)$, where $p_{s}^{\text {ins }}$ is the ratio of the number of successful test solvent particle insertions to the total number of attempted test solvent particle insertions. The bulk solvent pressure and chemical potential are obtained from the Carnahan-Starling equation of state for hard-spheres, ${ }^{23}$ and are given by

$$
\frac{P \sigma^{3}}{k_{B} T}=\frac{6 \eta}{\pi} \frac{\left(1+\eta+\eta^{2}-\eta^{3}\right)}{(1-\eta)^{3}}
$$

and

$$
\mu=\ln \left(\frac{6 \eta}{\pi}\right)+\frac{8 \eta-9 \eta^{2}+3 \eta^{3}}{(1-\eta)^{3}},
$$

where the first term on the right is the ideal contribution to the chemical potential, and the second term is the excess contribution to the chemical potential (over the ideal).

The approach of the system towards equilibrium is extremely slow, particularly at high densities and for large systems. This is partly due to the intrinsically slow relaxation of crosslinked systems, and partly due to the technique used for the volume changes. The latter constitutes a bottleneck for large systems since the positions and collision times of all the particles have to be recalculated following a successful volume change move, and the neighbor lists and time lists have to be reset. As a result, the above volume change method becomes unsuitable for systems larger than about 12000 segments. To give an approximate idea of the CPU time for these simulations, the generation of each simulation point (corresponding to a particular value of pressure and chemical potential) for our 20-mer and 35-mer networks (of 90 chains each) containing between 750 and 10000 solvent particles, required between 150 and $450 \mathrm{CPU}$ hours on a cluster of DEC Alphas with CPU speeds ranging from 125 to $500 \mathrm{MHz}$.

The question arises as to whether our combined DMD-MC technique satisfies the detailed balance condition,

$$
p_{i} p_{i j}=p_{j} p_{j i}
$$

where $p_{i}$ and $p_{j}$ represent the probabilities of the system being in state $i$ and $j$, respectively, and $p_{i j}$ and $p_{j i}$ represent the transition probabilities from state $i$ to $j$, and state $j$ to $i$, respectively. The purely Monte Carlo based techniques for phase equilibria are known to satisfy the above condition, provided that the three types of moves (particle displacements, volume changes and particle insertion/deletion) are carried out in a random order (although the total numbers of each type of move can be preset).$^{38}$ In our method, although the three types of moves are carried out in a random order, the necessity of incorporating the DMD method into the MC algorithm without introducing any biases associated with the unequal DMD time step has necessitated the carrying out of a few DMD particle displacement events between MC moves. These DMD events may cause the detailed balance condition [Eq. (12)] not to be fulfilled for the following reason: consider that the system is in state $i$, before an $\mathrm{MC}$ move (say, a volume change) is attempted. The MC move is attempted, is successful in accordance with the condition stated in Eq. (6) and the system is now in state, $j$. In order for the detailed balance condition [Eq. (12)] to be satisfied, the ratio of the transition probabilities, $p_{j i}(j \rightarrow i) / p_{i j}(i \rightarrow j)$, must be equal to the ratio, $p_{i} / p_{j}$, i.e., Eq. (6) must be the sole criterion determining whether the system can go back to state, $i$ from state $j$. If a few DMD particle displacements must occur before a second MC move is attempted, then the transition probability does not depend solely on Eq. (6) but also on whether the system has moved up to the next time step after executing an MC move. Hence the detailed balance condition is not strictly fulfilled. In our systems, the detailed balance condition will be fulfilled if the time step for the MC move is chosen to be sufficiently small so that no DMD events occur between attempted MC moves. In that case, the transition probabilities depend only on the criteria for volume changes and particle insertions/deletions, and the detailed balance condition is fulfilled. This is the case for our smaller systems. For our larger systems however, the choice of a small MC time step causes a very large increase in the total simulation time, and so we have allowed a few DMD events ( 0 to 4 ) to take place between the MC moves. As a result, the microscopic reversibility condition is not satisfied at every step of the process. We must keep in mind that the fulfillment of the detailed balance condition is a very strong condition $^{36,38}$ for the system to reach equilibrium. Previous studies using hybrid MC-MD ${ }^{35}$ techniques that do not strictly satisfy the detailed balance condition have been carried out and have resulted in adequate sampling of the configuration space and the correct equilibrium distributions. In our work, despite the drawback of not strictly fulfilling the detailed balance condition, the complexity of network simu- 
lations is such that a combined MC-MD technique such as ours has proved very useful in providing insights into the phase behavior of these systems.

\section{THEORY}

In this section, we extend our previously derived equation of state $^{2}$ for dry athermal networks to the case of network-solvent mixtures, i.e., gels. The basis for our dry network equation of state is the idea (James and $\mathrm{Guth}^{39}$ ) that the free energy of a network, $A_{\text {network }}(\eta)$, at any packing fraction, $\eta$, can be taken to be the sum of a liquidlike contribution, $A_{\text {liq }}(\eta)$, which is associated with the interatomic repulsive forces between the network monomers, and an elastic contribution, $A_{\text {elastic }}(\eta)$, which is associated with the retractive force exerted by the network chains opposing network deformation, i.e.,

$$
A_{\text {network }}(\eta)=A_{\text {liq }}(\eta)+A_{\text {elastic }}(\eta) .
$$

In our previous work, ${ }^{2}$ the liquidlike contribution is given by the free energy of a dendritic reference polymer which has the same excluded volume as the network, and the elastic contribution is given by a modified version of the affine model for rubberlike elasticity.

In the case of network-solvent mixtures (gels), the free energy of a gel is generally calculated using an approach which is analogous to that described above for dry networks, i.e., the gel free energy, $A_{\text {gel }}\left(\eta_{\text {gel }}, x\right)$, can be evaluated as the sum of a liquidlike contribution, $A_{\text {mix }}\left(\eta_{\text {gel }}, x\right)$, which is given by the free energy of a reference-polymer/solvent mixture, and an elastic contribution, $A_{\text {elastic }}\left(\eta_{\text {gel }}^{*}, 0\right)$, which has the same form as the dry network elastic free energy contribution. The liquidlike contribution to the gel free energy is a function of the gel packing fraction, $\eta_{\text {gel }}$, and the solvent mole fraction, $x$, while the elastic free energy is a function of the solvent-free gel packing fraction alone, $\eta_{\text {gel }}^{*}$, where $\eta_{\text {gel }}^{*}$ $\equiv \eta$ for the equivalent dry network (i.e., a dry network having the same number of network segments and the same volume as the gel). Thus the gel free energy is

$$
A_{\text {gel }}\left(\eta_{\text {gel }}, x\right)=A_{\text {mix }}\left(\eta_{\text {gel }}, x\right)+A_{\text {elastic }}\left(\eta_{\text {gel }}^{*}, 0\right) .
$$

In the following sections, the development of analytical expressions for the liquidlike and elastic contributions to the gel free energy, $A_{\text {mix }}\left(\eta_{\text {gel }}, x\right)$, and $A_{\text {elastic }}\left(\eta_{\text {gel }}^{*}, 0\right)$, is described. The analytical form for the gel free energy is then employed to predict the gel properties at swelling equilibria, i.e., the solvent fraction and packing fraction of the gel when it attains mechanical and chemical equilibrium in an external solvent at a given pressure and chemical potential. Since the development of the expression for the gel free energy is based on extending our equation of state for solvent-free networks to the case of mixtures, we begin with an outline of our theory for dry networks.

\section{A. Networks}

In this section, we outline our approach to calculating the elastic and liquidlike contributions to the free energy of dry networks (details are given in Ref. ${ }^{2}$ ). The network is modelled as a set of interpenetrating dendrimers, ${ }^{2}$ with each

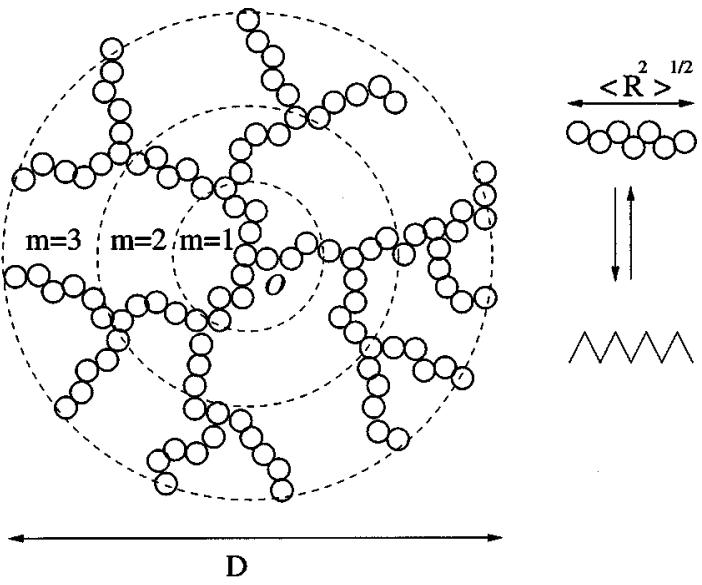

FIG. 1. Model of a single dendrimer in a network.

branch of a dendrimer representing an ideal, Gaussian network chain of length $n$. The dendrimers have the same functionality, $f$ as the network. Figure 1 is an example of a single such dendritic structure. It consists of concentric layers of branches or chains, and the total number of layers is called the rank of the network. The first layer of the dendrimer contains $f$ chains, the second layer contains $f(f-1)$ chains, the third layer contains $f(f-1)^{2}$ chains, and so on. The total number of chains in a dendrimer of rank $m$ is given by

$$
n_{\mathrm{den}}=f\left[\frac{(f-1)^{m}-1}{(f-2)}\right] \text {. }
$$

The elastic contribution to the network Helmholtz free energy is calculated by invoking the analogy between an ideal network chain and a classical elastic spring, ${ }^{40}$ such that the spring constant of the ideal network chain is given by, $k_{\text {chain }}=3 k_{B} T /\left\langle R_{0}^{2}\right\rangle$, where $\left\langle R_{0}^{2}\right\rangle=n l^{2}$ is the mean-square end-to-end distance of the ideal chain. The effective spring constant of a dendrimer molecule, $k_{\text {dendrimer }}$, composed of $n_{\text {den }}$ chains can be calculated in terms of the spring constant of a single chain, i.e., $k_{\text {dendrimer }}=k_{\text {chain }} f(f-2)[(f$ $\left.-1)^{(m-1)} /(f-1)^{m}-1\right]$. The network chain-elastic spring analogy can be carried further to obtain the elastic free energy of the dendrimer as, $A_{\text {dendrimer }}=k_{\text {dendrimer }} D^{2} / 2$, where $D^{2}$ is the squared spatial dimension or diameter of the dendrimer and is given by $D^{2}=2 m\left\langle R^{2}\right\rangle$, where $\left\langle R^{2}\right\rangle$ is the meansquared end-to-end distance of a dendrimer chain. When the network is deformed from an original volume $V_{0}$ to a final volume, $V=\lambda^{3} V_{0}$, where $\lambda$ is the deformation ratio, the dendrimers are assumed to deform affinely, that is, $D^{2}=\lambda^{2} D_{0}^{2}$, where $D_{0}^{2}=2 m \lambda^{2}\left\langle R_{0}^{2}\right\rangle$ denotes the mean-squared spatial dimension of the undeformed dendrimer.

The elastic component of the reduced pressure of an $f$-functional dendrimer-network of rank $m$, can be written $a^{2}$

$$
\begin{aligned}
\frac{P_{\text {elastic }}(\eta) \sigma^{3}}{k_{B} T} & =-\frac{\partial N_{D} A_{\text {dendrimer }}(\eta)}{\partial V} \\
& =-\frac{12 \eta_{0}}{\pi n} m(f-2)^{2}\left[\frac{(f-1)^{(m-1)}}{\left((f-1)^{m}-1\right)^{2}}\right]\left(\frac{\eta}{\eta_{0}}\right)^{1 / 3},
\end{aligned}
$$


where $N_{D}$ is the number of dendrimers in the network, and $\eta_{0}$ is the packing fraction of the undeformed network. To determine $\eta_{0}$, we must first define the undeformed state of the network. In our simulations, we started with networks at a packing fraction of $\eta=0.45$ and expanded them to successively lower packing fractions of $\eta=0.35,0.25, \ldots, 0.05$. Thus the network at $\eta=0.45$ is the starting point for all expansions of the system. At this packing fraction (i.e., $\eta=0.45$ ) we found that the mean-squared end-to-end distance of the network chains is equal to the mean-squared end-to-end distance of uncrosslinked system chains at the same packing fraction (shown in Ref. 2). Hence the configurational entropy of the network chains at a packing fraction of 0.45 is approximately the same as that of the equivalent uncrosslinked system chains at the same packing fraction. At any lower packing fraction $(\eta<0.45)$, the mean-squared end-to-end distance of the network chains is greater than the mean-squared end-to-end distance of uncrosslinked system chains, and the configurational entropy of the network chains is lower than that of the uncrosslinked system chains at that packing fraction. Based on this, the network is considered to be undeformed at the initial packing fraction of $\eta_{0}=0.45$ and to be deformed at all successive lower packing fractions. The deformation ratio of a network at any packing fraction $\eta \leqslant \eta_{0}$ can now be defined as

$$
\lambda=\lambda_{x}=\lambda_{y}=\lambda_{z}=\left[\frac{\eta_{0}}{\eta}\right]^{1 / 3}=\left[\frac{0.45}{\eta}\right]^{1 / 3}=\left[\frac{V}{V_{0}}\right]^{1 / 3} .
$$

The liquidlike contribution to the network pressure, $P_{\text {liq }}^{\text {net }}$, is obtained using an approach based on the generalized Flory-Dimer (GF-D) theory of Hall and co-workers. ${ }^{41-43}$ To evaluate $P_{\text {liq }}^{\text {net }}$, we postulate that $P_{\text {liq }}^{\text {net }}$ is equal to the pressure of a reference polymer having the same excluded volume as the network. For our dendrimer-network model, the reference polymer is simply a fluid of $f$-functional dendrimers of rank $m$. The compressibility factor of the dendrimer fluid is given $b y^{2}$

$$
\begin{aligned}
Z_{\mathrm{GF}-\mathrm{D}}^{\mathrm{net}}(\eta, f)= & {\left[\frac{v_{\text {dendrimer }}(n, m, f)-v_{e}(1)}{v_{e}(2)-v_{e}(1)}\right] Z_{2}(\eta) } \\
& -\left[\frac{v_{\text {dendrimer }}(n, m, f)-v_{e}(2)}{v_{e}(2)-v_{e}(1)}\right] Z_{1}(\eta),
\end{aligned}
$$

where $Z_{1}(\eta)$ and $Z_{2}(\eta)$ are the compressibility factors of fluids composed of hard sphere monomers and dimers, respectively, at the same packing fraction as the dendrimer fluid, $v_{\text {dendrimer }}(n, m, f)$ is the excluded volume of a dendrimer of rank $m$ and functionality, $f$, and $v_{e}(1)$ and $v_{e}(2)$ are the monomer and dimer excluded volumes, where $v_{e}(1)=(4 \pi / 3) \sigma^{3}$ and $v_{e}(2)=(9 \pi / 4) \sigma^{3}$.

The excluded volume of the dendrimer is obtained by treating the dendrimer fluid as a set of $f$-functional starcenters connected by linear arms (as shown in Fig. 1), and is given by

$$
\begin{aligned}
v_{\text {dendrimer }}(n, m, f)= & {\left[1+\frac{f(f-1)^{(m-1)}-1}{(f-2)}\right] v_{\text {center }}(f)+(n-4) } \\
& \times\left[f \frac{\left((f-1)^{m-1}-1\right)}{(f-2)}\right]\left(v_{e}(3)-v_{e}(2)\right) \\
& +2 f(f-1)^{(m-1)}\left(v_{e}(3)-v_{e}(2)\right)
\end{aligned}
$$

where $v_{\text {center }}(f)$ is the excluded volume of the star center, and $v_{e}(3)=9.82605 \sigma^{3}$ is the excluded volume of a trimer. The excluded volume of the star center is calculated using a Monte Carlo simulation technique following that of Alejandro and Chapela ${ }^{44}$ and Yethiraj and Hall; ${ }^{45}$ for a trifunctional network $(f=3), v_{\text {center }}(3)=18.01 \sigma \pm 0.05 \sigma$, and for a tetrafunctional network $(f=4), v_{\text {center }}(4)=23.06 \sigma \pm 0.03 \sigma^{2}$.

The compressibility factor of the monomer fluid, $Z_{1}(\eta)$, is obtained using the Carnahan-Starling equation of state, ${ }^{23}$

$$
Z_{1}(\eta)=\frac{1+\eta+\eta^{2}-\eta^{3}}{(1-\eta)^{3}}
$$

and the compressibility factor of the dimer fluid is obtained using the Tildesley-Streett equation of state ${ }^{46}$

$$
Z_{2}(\eta)=\frac{1+a_{1} \eta+a_{2} \eta^{2}-a_{3} \eta^{3}}{(1-\eta)^{3}}
$$

where $a_{1}, a_{2}, a_{3}$ are constants which take the values, $a_{1}$ $=2.45696, a_{2}=4.10386$, and $a_{3}=3.75503$.

The liquidlike contribution to the network pressure is obtained from the relation, $P_{\text {liq }}^{\text {net }}(\eta) / k_{B} T \equiv Z_{\mathrm{GF}-\mathrm{D}}^{\text {net }} N_{D} / V$ $=Z_{\mathrm{GF}-\mathrm{D}}^{\mathrm{net}}(\eta, f)\left(6 \eta /\left(\pi \sigma^{3} n_{\mathrm{den}} n\right)\right)$.

The total reduced pressure of the solvent-free network is then given by the sum of the elastic pressure [Eq. (16)] and the liquidlike pressure [Eq. (18)]

$$
\begin{aligned}
\frac{P_{\text {network }}(\eta) \sigma^{3}}{k_{B} T}= & \frac{P_{\text {elastic }}(\eta) \sigma^{3}}{k_{B} T}+\frac{P_{\text {liq }}^{\text {net }}(\eta) \sigma^{3}}{k_{B} T} \\
= & -\frac{12 \eta_{0}}{\pi n} m(f-2)^{2}\left[\frac{(f-1)^{(m-1)}}{\left((f-1)^{m}-1\right)^{2}}\right] \\
& \times\left(\frac{\eta}{\eta_{0}}\right)^{1 / 3}+Z_{\mathrm{GF}-\mathrm{D}}^{\text {net }}(\eta, f) \\
& \times \frac{6 \eta}{n \pi}\left[\frac{(f-2)}{f\left((f-1)^{m}-1\right)}\right] .
\end{aligned}
$$

\section{B. Gel swelling}

In this section, we extend our equation of state for solvent-free networks to the case of gels. Our primary objective is to develop analytical expressions for the gel free energy, pressure, and chemical potential which can be used to predict the extent of swelling and the solvent uptake of swelled gels in equilibrium with a bulk hard-sphere solvent.

Mathematically the conditions for an athermal gel to be in swelling equilibrium with an athermal bulk solvent may be written as

$$
P_{\text {bulk }}=P_{\text {gel }}, \quad \mu_{\text {bulk }}^{s}=\mu_{\text {gel }}^{s},
$$

where $P^{\text {bulk }}$ and $P^{\text {gel }}$ represent the bulk solvent and gel phase pressures, $\mu_{\text {bulk }}^{s}$ represents the bulk solvent chemical poten- 
tial, and $\mu_{\text {gel }}^{s}$ represents the solvent chemical potential in the gel phase. We now develop analytical expressions for each of the terms in the above equation.

The pressure of an athermal gel (an athermal network swelled with athermal solvent) can be written ${ }^{21,22,5}$ in a form analogous to Eq. (22), as the sum of an elastic contribution, and a liquidlike contribution,

$$
\frac{P_{\mathrm{gel}}(\eta, x) \sigma^{3}}{k_{B} T}=\frac{P_{\mathrm{elastic}}\left(\eta^{*}, 0\right) \sigma^{3}}{k_{B} T}+\frac{P_{\mathrm{mix}}(\eta, x) \sigma^{3}}{k_{B} T} .
$$

The elastic contribution to the gel pressure, $P_{\text {elastic }}\left(\eta^{*}, 0\right)$, is calculated on a solvent-free basis, and has exactly the same form as described in the preceding section for dry networks [cf. Eq. (16)]. The solvent-free packing fraction of the network, $\eta^{*}$, is given by $\eta^{*}=\pi N_{D}\left(n_{\mathrm{den}} n\right) \sigma^{3} / 6 V$, where $N_{\mathrm{D}}$ is the number of dendrimer molecules, and $\left(n_{\mathrm{den}} n\right)$ is the number of segments in each dendrimer molecule; $\eta^{*}$ can also be written in terms of the solvent mole fraction and gel packing fraction as $\eta^{*}=\eta\left[(1-x)\left(n_{\operatorname{den}} n\right)\right] /\left[x+(1-x)\left(n_{\operatorname{den}} n\right)\right]$, where $x$ is the solvent mole fraction in the gel.

The liquidlike contribution to the gel pressure, $P_{\text {mix }}(\eta, x)$, is obtained from the thermodynamic properties of a mixture of dendrimer and solvent molecules at the same packing fraction and composition as the gel, and is evaluated using a simple conformal-solution-type equation, following Honnell and Hall. ${ }^{25}$ For a mixture of $N_{\mathrm{D}}$ dendrimer molecules and $N_{s}$ solvent molecules having the same segment diameter, the compressibility factor of the mixture, $Z_{\text {mix }}$ $\equiv P_{\text {mix }} V / k_{B} T\left(N_{\mathrm{D}}+N_{s}\right)$, is given by the mole fraction average of the GF-D compressibility factors of the pure, constituent species evaluated at the packing fraction of the mixture,

$$
Z_{\text {mix }}(\eta, x)=x Z_{s}(\eta)+(1-x) Z_{\mathrm{GF}-\mathrm{D}}^{\mathrm{net}}(\eta),
$$

where $Z_{s}(\eta)$ is the compressibility factor of the solvent, and $Z_{\mathrm{GF}-\mathrm{D}}^{\text {net }}(\eta)$ is the compressibility factor of the dendrimermolecule reference polymer system.

The total reduced pressure of the gel is then given by the sum of the elastic and liquidlike contributions to the pressure,

$$
\begin{aligned}
\frac{\left.P_{\text {gel }}(\eta, x)\right] \sigma^{3}}{k_{B} T}= & \frac{P_{\text {elastic }}\left(\eta^{*}, 0\right) \sigma^{3}}{k_{B} T}+\frac{P_{\text {mix }}(\eta, x) \sigma^{3}}{k_{B} T} \\
= & -\frac{12 \eta_{0}}{\pi n} m(f-2)^{2}\left[\frac{(f-1)^{(m-1)}}{\left((f-1)^{m}-1\right)^{2}}\right] \\
& \times\left(\frac{\eta^{*}}{\eta_{0}}\right)^{1 / 3}+Z_{\text {mix }}(\eta, x) \frac{6 \eta}{\pi} \\
& \times\left[n \frac{(f-2)}{\left(f\left((f-1)^{m}-1\right)(1-x)+x(f-2)\right)}\right] .
\end{aligned}
$$

The last term in the above equation is obtained from the expression $P_{\text {mix }}=Z_{\text {mix }}\left(N_{\mathrm{D}}+N_{s}\right) / V$ where $N_{\mathrm{D}}$ and $N_{s}$ are rewritten in terms of the packing fraction, $\eta$, and the solvent mole fraction, $x$, by using the definitions $\eta$ $=(\pi / 6) \sigma^{3}\left(N_{\mathrm{D}} n_{\mathrm{den}} n+N_{s}\right) / V$ and $x=N_{s} /\left(N_{\mathrm{D}}+N_{s}\right)$. We now look at how Eq. (23) and Eq. (26) can be used to predict the phase equilibrium properties of the swelled gel.

Since the bulk solvent in our system is a pure hardsphere fluid, its thermodynamic properties can be obtained from the Carnahan-Starling equation of state. ${ }^{23}$ The pressure of the bulk solvent is given by Eq. (20), evaluated at the bulk solvent packing fraction, $\eta_{\text {bulk }}$. The chemical potential of the bulk solvent is given by Eq. (11) evaluated at the bulk solvent packing fraction. The thermodynamic properties of the bulk phase remain unchanged as the gel swells.

To obtain the gel properties (i.e., the packing fraction and solvent mole fraction) at which the swelling equilibrium conditions in Eq. (23) are satisfied, we must find how the gel phase pressure, $P_{\text {gel }}$ and solvent chemical potential, $\mu_{\text {gel }}^{s}$ depend on the packing fraction and mole fraction. The pressure of the gel phase can be obtained using Eq. (26). The chemical potential of the solvent in the gel phase can be evaluated if the Helmholtz free energy of the gel is known as a function of the solvent mole fraction, $x$, at constant $V$ and $N_{k}$, where $N_{k}$ is the number of molecules of other (nonsolvent) species present:

$$
\begin{aligned}
\frac{\mu_{\mathrm{gel}}^{s}}{k_{B} T} & =\frac{\mu_{\mathrm{id}, \mathrm{gel}}^{s}}{k_{B} T}+\frac{\mu_{\mathrm{ex}, \mathrm{gel}}^{s}}{k_{B} T} \\
& =\left.\frac{\partial A_{\mathrm{id}, \mathrm{gel}} / k_{B} T}{\partial N_{s}}\right|_{V, T, N_{k}}+\left.\frac{\partial A_{\mathrm{ex}, \mathrm{gel}} / k_{B} T}{\partial N_{s}}\right|_{V, T, N_{k}},
\end{aligned}
$$

where $A_{\text {id,gel }}$ and $A_{\text {ex,gel }}$ are the ideal gas and excess contributions to the gel free energy. In our system, since we have a single-component solvent, $N_{k}$ is equal to the number of dendrimer molecules, which always remains constant during the swelling process (since the solvent is the only migrant species).

To calculate the solvent chemical potential, we must calculate the total Helmholtz free energy $A_{\text {id, gel }}+A_{\text {ex,gel }}$ as a function of the solvent mole fraction, $x$, at constant volume, $V$. From Eq. (14), we already know that the Helmholtz free energy of the gel, $A_{\text {gel }}(\eta, x)$, is the sum of the elastic contribution, $A_{\text {elastic }}\left(\eta^{*}, 0\right)$, and the liquidlike contribution $A_{\text {mix }}(\eta, x)$. The liquidlike contribution to the free energy of the gel can be calculated as the sum of an ideal component, $A_{\text {id,mix }}$ and an excess component, $A_{\text {ex,mix }}$. The ideal component of the Helmholtz free energy of the gel, $A_{\text {id,gel }}$, is equal to the ideal component of the liquidlike contribution to the gel free energy, $A_{\text {id,mix }}$, because the elastic contribution to the gel free energy does not contain an ideal component, and because $A_{\text {id,mix }}(\eta, x)$ is evaluated at the same packing fraction and mole fraction as the gel. Hence, the excess Helmholtz free energy of the gel, $A_{\text {ex,gel }}(\eta, x)$ is the sum of the excess component of the liquidlike free energy contribution, $A_{\text {ex,mix }}(\eta, x)$, and the elastic contribution, $A_{\text {elastic }}\left(\eta^{*}, 0\right)$. We can now use our expression for $Z_{\text {mix }}$ (Eq. 25) to evaluate the excess component of the liquidlike free energy contribution, 


$$
\begin{aligned}
\frac{A_{\mathrm{ex}, \text { mix }}(\eta, x)}{\left(N_{\mathrm{D}}+N_{s}\right) k_{B} T}= & \int_{0}^{\eta}\left[Z_{\mathrm{mix}}\left(\eta^{\prime}, x\right)-1\right] \frac{d \eta^{\prime}}{\eta^{\prime}} \\
= & \int_{0}^{\eta}\left[\left(x Z_{s}\left(\eta^{\prime}\right)+(1-x)\right.\right. \\
& \left.\left.\times Z_{\mathrm{GF}-\mathrm{D}}^{\mathrm{net}}\left(\eta^{\prime}\right)\right)-1\right] \frac{d \eta^{\prime}}{\eta^{\prime}} \\
= & x \int_{0}^{\eta}\left(Z_{s}\left(\eta^{\prime}\right)-1\right) \frac{d \eta^{\prime}}{\eta^{\prime}}+(1-x) \\
& \times \int_{0}^{\eta}\left(Z_{\mathrm{GF}-\mathrm{D}}^{\mathrm{net}}\left(\eta^{\prime}\right)-1\right) \frac{d \eta^{\prime}}{\eta^{\prime}} .
\end{aligned}
$$

For a pure hard-sphere solvent, $Z_{s}\left(\eta^{\prime}\right)$ is given by Eq. (20). For $Z_{\mathrm{GF}-\mathrm{D}}^{\text {net }}\left(\eta^{\prime}\right)$, we use the expression given by Eq. (18), and obtain an analytical expression for the excess component of the liquidlike free energy

$$
\begin{aligned}
\frac{A_{\mathrm{ex}, \text { mix }}(\eta, x)}{\left(N_{\mathrm{D}}+N s\right) k_{B} T}= & x\left[\frac{4 \eta-3 \eta^{2}}{(1-\eta)^{2}}\right]+(1-x) \\
& \times\left[\frac{1}{(1-\eta)^{2}}\left(\frac{A_{1}+A_{2}+A_{3}}{2}\right)\right. \\
& -\frac{1}{(1-\eta)}\left(A_{2}+2 A_{3}\right)-A_{3} \ln (1-\eta) \\
& \left.-\left(\frac{A_{1}+A_{2}-3 A_{3}}{2}\right)\right]
\end{aligned}
$$

where $A_{1}, A_{2}$ and $A_{3}$ are constants given by

$$
\begin{aligned}
& A_{1}=\left(a_{1}-1\right) Y_{n}+\left(a_{1}+3\right), \\
& A_{2}=\left(a_{2}-1\right) Y_{n}+\left(a_{2}-3\right), \\
& A_{3}=\left(1-a_{3}\right) Y_{n}+\left(1-a_{3}\right),
\end{aligned}
$$

and $a_{1}, a_{2}, a_{3}$ are constants from the Tildesley-Streett equation of state [Eq. (21)], and $Y_{n}=\left(v_{\text {dendrimer }}(n, m, f)\right.$ $\left.-v_{e}(1)\right) /\left(v_{e}(2)-v_{e}(1)\right)$. The elastic component of the excess free energy of the gel is given $b^{2}$

$A_{\text {elastic }}\left(\eta^{*}, 0\right)=3 k_{B} \operatorname{Tm} f(f-2)\left[\frac{(f-1)^{(m-1)}}{(f-1)^{m}-1}\right]\left(\frac{\eta_{0}}{\eta^{*}}\right)^{2 / 3}$.

The chemical potential of the solvent in the gel phase is then

$$
\mu_{\mathrm{gel}}^{s}=\frac{\partial}{\partial N_{s}}\left[A_{\mathrm{id}, \mathrm{mix}}\right]_{V, N_{k}}+\frac{\partial}{\partial N_{s}}\left[A_{\mathrm{ex}, \text { mix }}+A_{\text {elastic }}\right]_{V, N_{k}} .
$$

Since the elastic free energy contribution [Eq. (31)] depends only the volume change of the network during swelling, and is calculated on a solvent free basis, the term, $\partial A_{\text {elastic }} / \partial N_{s}$ is zero under constant volume conditions. Hence Eq. (32) can be rewritten as

$$
\mu_{\mathrm{gel}}^{s}=\frac{\partial}{\partial N_{s}}\left[A_{\mathrm{id}, \mathrm{mix}}\right]_{V, N_{k}}+\frac{\partial}{\partial N_{s}}\left[A_{\mathrm{ex}, \mathrm{mix}}\right]_{V, N_{k}} .
$$

Expressing $x$ and $\eta$ in terms of $N_{s}$, i.e., $x=N_{s} /\left(N_{s}+N_{\mathrm{D}}\right)$, and $\eta=\pi\left[N_{s}+N_{\mathrm{D}}\left(n_{\mathrm{den}} n\right)\right] / 6 V$, the term $A_{\mathrm{ex}, \text { mix }}$ can be re- written in terms of the single variable, $N_{s}$. Taking the derivative of $A_{\text {id,mix }} \quad\left[\right.$ where $A_{\text {id,mix }}=k_{B} T\left(N_{s}+N_{\mathrm{D}}\right) \ln \left[\left(N_{s}\right.\right.$ $\left.\left.\left.+N_{\mathrm{D}}\right) / V\right]-1\right]$ and $A_{\text {ex,mix }}$ with respect to $N_{s}$ then yields

$$
\begin{aligned}
\mu_{\mathrm{gel}}^{s}= & \ln \left(\rho_{s}\right)+\left[\phi \frac{\eta(4-2 \eta)}{(1-\eta)^{3}}+\frac{4 \eta-3 \eta^{2}}{(1-\eta)^{2}}\right]+\frac{(1-\phi) \eta}{\left(n_{\mathrm{den}} n\right)} \\
& \times\left[\frac{\left(A_{1}+A_{2}+A_{3}\right)}{(1-\eta)^{3}}-\frac{\left(A_{2}+2 A_{3}\right)}{(1-\eta)^{2}}+\frac{A_{3}}{(1-\eta)}\right],
\end{aligned}
$$

where $\rho_{s}=N_{s} / V$ is the number density of the solvent molecules in the gel, and $\phi=\left(n_{\mathrm{den}} n\right) x /\left[(1-x)+\left(n_{\mathrm{den}} n\right) x\right]$ is the solvent site fraction.

Since we now know the gel pressure and the solvent chemical potential that appear in Eq. (23) in terms of the packing fraction and mole fraction, we can proceed with our phase equilibria calculations. Our aim is to calculate the value of the solvent mole fraction, $x$, and the gel packing fraction, $\eta$, at which the gel pressure and the solvent chemical potential in the gel equal the predetermined values of the bulk solvent pressure and chemical potential. This is done by adopting the method and numerical techniques of Kenkare et al., ${ }^{47}$ as outlined below. At predetermined values of the bulk solvent pressure, $P_{\text {bulk }}$ we iterate on $\eta$ at each value of $x$ (in increments of 0.00001 ), until we find the packing fraction at which the evaluated pressure, $P_{\text {gel }}$ approaches $P_{\text {bulk }}$ within some tolerance $\delta \leqslant 10^{-6}$. This gives us a curve for $\eta$ versus $x$ at which $P_{\text {gel }}$ equals $P_{\text {bulk }}$. In order to find out which value of $\eta(x)$ satisfies the criterion, $\mu_{\text {gel }}^{s}=\mu_{\text {bulk }}^{s}$ we then use Eq. (34) to evaluate the solvent chemical potential as a function of $x$. When $\mu_{\text {gel }}^{s}$ equals $\mu_{\text {bulk }}^{s}$ within some tolerance $\delta_{1}<10^{-3}$, the conditions for swelling equilibrium are satisfied. This method is used to calculate the gel packing fractions and solvent mole fractions over a range of bulk solvent pressures.

\section{RESULTS AND DISCUSSION}

In Tables III and IV we present simulation results for the thermodynamic properties of 20-mer and 35-mer trifunctional gels that are swelled to equilibrium in a hardsphere solvent. The tables show the reduced pressure and chemical potential of the bulk solvent phase, $P_{\text {bulk,CS }}^{*}$ and $\mu_{\text {bulk,CS }}^{s}$ (calculated using the Carnahan-Starling ${ }^{23}$ equation of state), and our simulation results for the reduced pressure and the solvent chemical potential of the gel phase, $P_{\text {gel }}^{*}$ and $\mu_{\text {gel }}^{s}$, the packing fraction of the gel phase, $\eta=\pi\left(N_{s}+N_{c} n\right) \sigma^{3} / 6 V$, the solvent site fraction, $\phi=N_{s} /\left(N_{s}+N_{c} n\right)$, the number of solvent molecules in the gel, $N_{s}$, and the total number of segments (network and solvent) in the system, $N_{t}$. The estimated uncertainties in the gel phase properties were obtained by letting each simulation continue for six or more runs of 50-300 million collisions each (depending on the system size) after the equilibration runs, and calculating the standard deviation from the properties measured during these runs. We found generally good agreement within the error estimates between the pressure and chemical potential in the bulk solvent phase and our simulation results for the pressure and solvent chemical potential in the gel phase, thus confirming that the gels had reached swelling equilibrium. Data was obtained for pressures ranging from $P^{*}=0.1$ to $P^{*}$ 
TABLE III. Equilibrium data for the 20-mer gel. Values for the bulk solvent pressure, $P_{\text {bulk,CS }}^{*}$, and chemical potential, $\mu_{\text {bulk,CS }}^{s}$, calculated from the Carnahan-Starling (Ref. 23) (CS) equation of state for hard spheres, and our simulation results for the gel pressure, $P_{\text {gel }}^{*}$, solvent chemical potential, $\mu_{\text {gel }}^{s}$, gel packing fraction, $\eta$, solvent site fraction, $\phi$, the number of solvent molecules in the gel, $N_{s}$, and the total number of segments in the gel, $N_{t}$. The values in parentheses indicate the uncertainty in the last digit; for instance a value of 4.99(4) is equivalent to a value of $4.99 \pm 0.04$.

\begin{tabular}{cccccccc}
\hline \hline$P_{\text {bulk,CS }}^{*}$ & $\mu_{\text {bulk,CS }}^{s}$ & $P_{\text {gel }}^{*}$ & $\mu_{\text {gel }}^{s}$ & $\eta$ & $\phi$ & $N_{s}$ & $N_{t}$ \\
\hline 0.1 & -2.09 & $0.105(4)$ & $-2.05(3)$ & $0.106(2)$ & $0.286(4)$ & $721 \pm 13$ & $2521 \pm 13$ \\
0.5 & 0.219 & $0.501(3)$ & $0.216(40)$ & $0.180(1)$ & $0.570(1)$ & $2386 \pm 10$ & $4186 \pm 10$ \\
1.0 & 1.73 & $1.003(4)$ & $1.73(1)$ & $0.239(1)$ & $0.643(1)$ & $3242 \pm 15$ & $5024 \pm 15$ \\
3.0 & 5.49 & $2.98(2)$ & $5.48(4)$ & $0.351(1)$ & $0.698(1)$ & $4160 \pm 20$ & $5960 \pm 20$ \\
5.0 & 8.37 & $4.99(4)$ & $8.38(6)$ & $0.409(1)$ & $0.701(1)$ & $4220 \pm 20$ & $6020 \pm 20$ \\
\hline \hline
\end{tabular}

$=5.0$ for the 20-mer gels, and for pressures ranging from $P^{*}=0.1$ to $P^{*}=3.0$ for the 35 -mer gels. We were unable to obtain swelling data for the 35-mer network at pressures higher than $P^{*}=3.0$ owing to computational limitations associated with the large number of solvent molecules in the system (the 35-mer network at $P^{*}=3.0$ contained about 9300 solvent molecules in addition to 3150 network segments).

Figure 2 displays the reduced pressure versus packing fraction for a pure hard-sphere fluid (from the CarnahanStarling equation of state $^{23}$ ), a trifunctional 20-mer gel swelled to equilibrium in hard-sphere solvent (from our simulation results, cf. Table III), and a solvent-free trifunctional 20-mer network (from our previously published ${ }^{2}$ simulation results, cf. Table II). This plot helps us to compare the magnitude and trends in the gel properties with the magnitudes and trends in the pure solvent and network properties. The lines through the 20-mer gel and solvent-free network data show our theoretical predictions for the gel (from Sec. III B) and for the network [Eq. (22)]. We see that, at a given packing fraction, the pressure of the gel always lies between the pressures of the bulk-hard-sphere solvent and the pure network. The theoretical prediction for the gel packing fraction agrees very well with the simulation data.

Figure 3 shows the equilibrium values for the solvent site fraction, $\phi$, and the gel packing fraction, $\eta$, versus reduced gel pressure, $P_{\text {gel }}^{*}$, for the 20-mer and 35-mer networks. Both the solvent site fraction and the gel packing fraction are observed to increase with pressure. The solid lines show the predictions of our theory for the 20-mer network, and the dashed lines show the predictions of our theory for the 35-mer network. The numerical values for our predictions are shown in Tables V and VI. It is evident that the theoretical predictions for the packing fraction show excellent agreement with the simulation results. The theory also shows good agreement with the solvent site fraction data, although it overpredicts the solvent site fraction at high pressures for the 20-mer and the 35-mer networks. The reason for the overprediction of $\phi$ by our theory at high pressures is not completely clear; a possible explanation may be that the elastic term [Eq. (22)] performs poorly at high pressures, since the gel is highly deformed (expanded) and the network chains are considerably extended. As a result the chain vector distribution may deviate significantly from the Gaussian, causing an increase in the magnitude of the elastic contribution to the network pressure, and a decrease in the overall network pressure. A similar and possibly related phenomenon was observed by us in our previous work on the pressure-volume behavior of dry networks; we observed that when such networks were expanded to very large deformation ratios there was a sudden increase in the magnitude of the elastic component of the network pressure (which is negative), and our theory (being based on a Gaussian chain model) was less able to accurately predict the network pressure. Similarly, in highly swelled gels, the magnitude of the negative elastic contribution to the network pressure may be greater than is predicted by our theory, resulting in overprediction of the gel pressure.

From Fig. 3 and the values in Tables III and IV, we observe that the equilibrium gel packing fraction of the 35mer network is slightly lower than that of the 20-mer network at any given pressure, i.e., the pressure of the gel increases with increasing chain length at any given packing fraction. This trend is similar to that seen in dry networks and is accurately predicted by our theory. In an equivalent uncrosslinked system (in which the system pressure is given by the mixing contribution alone), the pressure decreases with increasing chain length. To understand why the gel pressure should increase with increasing chain length, it is necessary to recall that the magnitude of the negative elastic contribution to the gel pressure decreases with increasing chain length at a given packing fraction [cf. Eq. (16)]. This is

TABLE IV. Equilibrium data for the 35-mer gel. Notation as in Table III.

\begin{tabular}{llllcccc}
\hline \hline$P_{\text {bulk,CS }}^{*}$ & $\mu_{\text {bulk,CS }}^{s}$ & \multicolumn{1}{c}{$P_{\text {gel }}^{*}$} & \multicolumn{1}{c}{$\mu_{\text {gel }}^{s}$} & $\eta$ & $\phi$ & $N_{s}$ & $N_{t}$ \\
\hline 0.1 & -2.09 & $0.100(1)$ & $-2.12(2)$ & $0.095(1)$ & $0.325(3)$ & $1517 \pm 20$ & $4667 \pm 20$ \\
0.3 & -0.633 & 0.296 & -0.65 & $0.139(1)$ & $0.545(3)$ & $3775 \pm 45$ & $6925 \pm 45$ \\
1.0 & 1.73 & $1.01(1)$ & $1.74(1)$ & $0.236(1)$ & $0.675(1)$ & $6542 \pm 30$ & $9692 \pm 30$ \\
1.9 & 3.63 & 1.92 & 3.60 & $0.299(0)$ & $0.732(0)$ & $8600 \pm 5$ & $11750 \pm 5$ \\
2.94 & 5.42 & $2.98(2)$ & $5.48(4)$ & $0.345(1)$ & $0.747(1)$ & $9300 \pm 50$ & $12450 \pm 50$ \\
\hline \hline
\end{tabular}




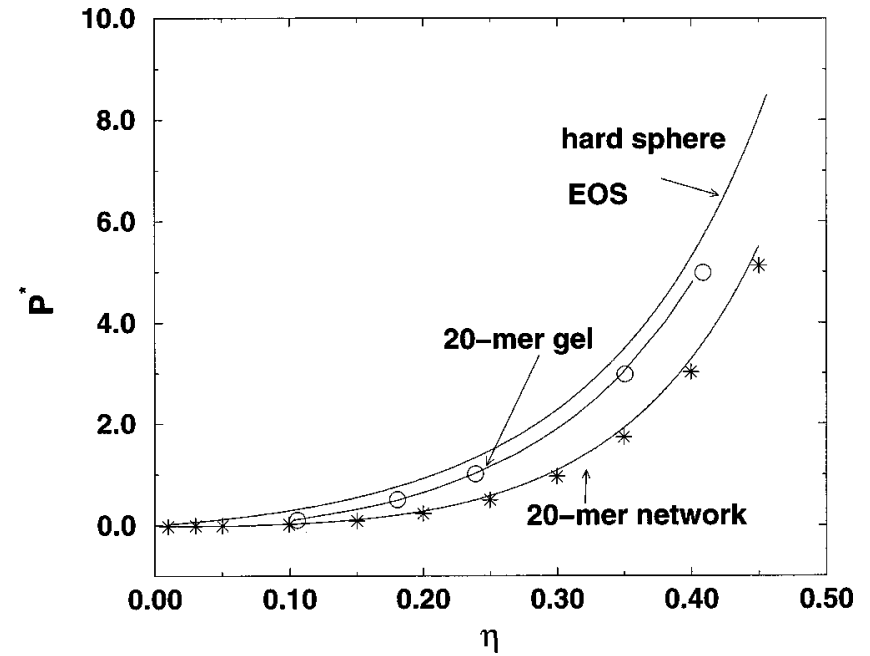

FIG. 2. Reduced pressure, $P^{*}=P \sigma^{3} / k_{B} T$, versus packing fraction, $\eta$, for (1) a pure hard-sphere fluid, (2) a 20-mer trifunctional gel, and (3) a 20-mer solvent-free trifunctional network. The lines through the gel and network data points represent our theoretical predictions.

because the elastic contribution to the gel pressure arises from the retractive force exerted by the chains opposing network deformation, which in turn is related to the ratio of the end-to-end distance of the network chains to their maximum extended length. ${ }^{2}$ At a given packing fraction or deformation ratio, the ratio of the end-to-end distance of a network chain to its maximum extended length is greater for the chains in a short chain-length network than for the chains in a long chain-length network. Hence the short network chain undergoes a greater loss of configurational entropy than a long network chain at a given network deformation ratio, and exerts a correspondingly greater retractive force on the network. Thus, the magnitude of the retractive force exerted by the chains increases with decreasing chain length at a given deformation ratio. The corresponding negative elastic contri-

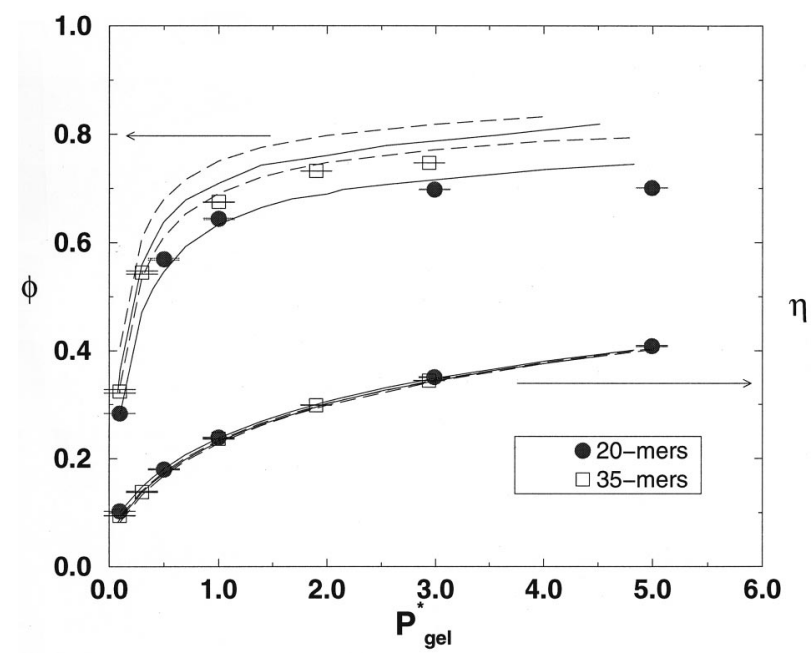

FIG. 3. Simulation results for the solvent site fraction, $\phi$, and the packing fraction, $\eta$, of the gel versus reduced pressure, $P^{*}=P_{\mathrm{gel}} \sigma^{3} / k_{B} T$ for the 20 -mer and 35-mer networks. The solid lines represent our theoretical predictions for the 20-mer network, and the dashed lines represent our theoretical predictions for the 35 -mer network.
TABLE V. Comparison between our simulation results and our theoretical prediction for the 20-mer gel. Simulation data for the gel packing fraction, $\eta$, and solvent site fraction, $\phi$, and our theoretical predictions, $\eta_{\text {theory }}$ and $\phi_{\text {theory }}$.

\begin{tabular}{cccccc}
\hline \hline$P_{\text {bulk,CS }}^{*}$ & $\mu_{\text {bulk,CS }}^{s}$ & $\eta$ & $\phi$ & $\eta_{\text {theory }}$ & $\phi_{\text {theory }}$ \\
\hline 0.1 & -2.09 & $0.106(2)$ & $0.286(4)$ & 0.102 & 0.283 \\
0.5 & 0.219 & $0.180(1)$ & $0.570(1)$ & 0.180 & 0.551 \\
1.0 & 1.73 & $0.239(1)$ & $0.643(1)$ & 0.237 & 0.634 \\
3.0 & 5.49 & $0.351(1)$ & $0.698(1)$ & 0.349 & 0.716 \\
5.0 & 8.37 & $0.409(1)$ & $0.701(1)$ & 0.405 & 0.745 \\
\hline \hline
\end{tabular}

bution to the pressure increases in magnitude also, causing the total pressure of the gel to decrease with decreasing chain length.

From Fig. 3 and Tables III and IV, we also observe that the equilibrium value of the solvent site fraction increases with increasing chain length at a given pressure and bulk solvent chemical potential. We can understand why this is so by returning to the chemical potential equilibrium condition [Eqs. (23) and (27)].

$$
\mu_{\mathrm{bulk}}^{s}=\mu_{\mathrm{gel}}^{s}=\mu_{\mathrm{id}, \mathrm{gel}}^{s}+\mu_{\mathrm{ex}, \mathrm{gel}}^{s},
$$

where $\mu_{\mathrm{id}, \mathrm{gel}}^{s}$ and $\mu_{\mathrm{ex}, \mathrm{gel}}^{s}$ are the ideal and excess components of the chemical potential of the solvent in the gel. At a given value of the bulk solvent pressure and chemical potential, the solvent chemical potentials in a 20-mer and 35-mer gel at swelling equilibrium are both equal to $\mu_{\text {bulk }}^{s}$ although the magnitudes of the individual ideal and excess chemical potential contributions are not the same. The ideal component of the solvent chemical potential is given by $\mu_{\mathrm{id}, \mathrm{sel}}^{s}$ $\equiv k_{B} T \log \left(\rho_{s}\right)=k_{B} T \log (\eta \phi)$, where $\rho_{s}$ is the number density of the solvent molecules. The excess component of the solvent chemical potential is calculated using the relation $\mu_{\text {ex,gel }}^{s} \equiv-k_{B} T \log \left(\rho_{s}^{\text {ins }}\right)$, where $p_{s}^{\text {ins }}$ is the probability of inserting a test solvent molecule into the gel. Now we saw earlier that at a given bulk solvent pressure or chemical potential, the equilibrium packing fraction of the gel decreases with increasing chain length, i.e., the packing fraction of the 20-mer gel at swelling equilibrium is higher than the packing fraction of the 35-mer network under the same conditions. Therefore we expect that it will be more difficult to insert a solvent molecule into the 20-mer gel than into the 35-mer gel at that bulk pressure or chemical potential, and therefore, $p_{s}^{\text {ins }}(20)<p_{s}^{\text {ins }}(35)$. Hence the excess solvent chemical potential of the 20-mer gel will be higher than that of the 35mer gel, i.e., $\mu_{\text {ex,gel }}^{s}(20)>\mu_{\text {ex,gel }}^{s}(35)$. Since both the 20-mer

TABLE VI. Comparison between our simulation results and our theoretical prediction for the 35-mer gel. Simulation data for the gel packing fraction, $\eta$, and solvent site fraction, $\phi$, and our theoretical predictions, $\eta_{\text {theory }}$ and $\phi_{\text {theory }}$.

\begin{tabular}{cccccc}
\hline \hline$P_{\text {bulk,CS }}^{*}$ & $\mu_{\text {bulk,CS }}^{s}$ & $\eta$ & $\phi$ & $\eta_{\text {theory }}$ & $\phi_{\text {theory }}$ \\
\hline 0.1 & -2.09 & $0.095(1)$ & $0.325(3)$ & 0.089 & 0.365 \\
0.3 & -0.633 & $0.139(1)$ & $0.545(3)$ & 0.136 & 0.559 \\
1.0 & 1.73 & $0.236(1)$ & $0.675(1)$ & 0.232 & 0.709 \\
1.9 & 3.63 & $0.299(0)$ & $0.732(0)$ & 0.295 & 0.760 \\
2.9 & 5.42 & $0.345(1)$ & $0.747(1)$ & 0.343 & 0.779 \\
\hline \hline
\end{tabular}


TABLE VII. NPT-ensemble simulation results for the evolution of the packing fraction, $\eta$, the deformation ratio, $\lambda$, and the chain mean-squared endend distance, $\left\langle R^{2}\right\rangle / \sigma^{2}$, for the 20-mer gel at various solvent site fractions, $\phi$, as the network swells to its equilibrium state at $P^{*}=0.5$ and $P^{*}=1.0$.

\begin{tabular}{lllcc}
\hline \hline$P^{*}$ & \multicolumn{1}{c}{$\phi$} & \multicolumn{1}{c}{$\eta$} & $\lambda$ & $\left\langle R^{2}\right\rangle / \sigma^{2}$ \\
\hline 0.5 & 0.0 & 0.250 & 1.216 & 41.12 \\
0.5 & 0.114 & 0.234 & 1.293 & 44.82 \\
0.5 & 0.205 & 0.222 & 1.367 & 48.24 \\
0.5 & 0.400 & 0.199 & 1.556 & 56.79 \\
0.5 & 0.567 & 0.179 & 1.798 & 68.15 \\
1.0 & 0.0 & 0.302 & 1.142 & 36.92 \\
1.0 & 0.114 & 0.293 & 1.200 & 40.08 \\
1.0 & 0.205 & 0.283 & 1.259 & 42.75 \\
1.0 & 0.283 & 0.275 & 1.316 & 45.31 \\
1.0 & 0.400 & 0.261 & 1.421 & 50.63 \\
1.0 & 0.645 & 0.239 & 1.743 & 63.98 \\
\hline \hline
\end{tabular}

and 35-mer gels are at the same total chemical potential, the ideal component of the 20-mer solvent chemical potential must be lower than that of the 35-mer, $\mu_{\text {id,gel }}^{s}(20)$ $<\mu_{\text {id,gel }}^{s}(35)$. Using the previously given expression for ideal chemical potential, we can write

$$
\begin{aligned}
k_{B} T \log [\eta(20) \phi(20)]< & k_{B} T \log [\eta(35) \phi(35)] \\
& \Rightarrow \frac{\phi(20)}{\phi(35)}<\frac{\eta(35)}{\eta(20)} .
\end{aligned}
$$

Since the packing fraction of the 35-mer gel is lower than the packing fraction of the 20 -mer gel, i.e., $\eta(35) / \eta(20)<1$, we conclude that $\phi(20) / \phi(35)<1$ at a given pressure and chemical potential, or that the solvent site fraction for the 20-mer is lower than that of the 35-mer. Hence the solvent site fraction decreases with chain length at constant pressure and chemical potential.

We investigate the change in thermodynamic properties of the gel during evolution of the system from a solvent-free network to a swollen gel in mechanical and chemical equilibrium with the bulk solvent by adopting the approach used by Escobedo and de Pablo. We conducted a set of simulations of the gel in which the gel pressure is held constant and equal to the bulk solvent pressure. Within this set of simulations at a given pressure, the solvent site fraction is increased incrementally from zero (dry network) to $\phi_{\text {equil }}$, the solvent site fraction at swelling equilibrium at that pressure. For each individual simulation run within the set, the solvent site fraction is kept constant. Two values of the bulk solvent pressure are chosen, $P_{\text {bulk }}^{*}=0.5$ and 1.0. Each simulation data point was obtained by starting with a dry network $(\phi=0)$ at a network pressure equal to one of the selected bulk solvent pressure values, inserting a fixed number of solvent molecules into the network, and letting the gel relax to its final volume keeping the pressure constant (an NPT simulation). Table VII displays our simulation results for the gel packing fraction for the two sets of runs conducted at $P^{*}=0.5$ and $P^{*}=1.0$, both for the 20-mer network. At each value of $\phi$, the table shows the gel packing fraction, $\eta$, the deformation ratio, $\lambda$, and the mean-squared end-to end distance of the network chains, $\left\langle R^{2}\right\rangle / \sigma^{2}$.

Figure 4 shows the simulation results for the gel packing

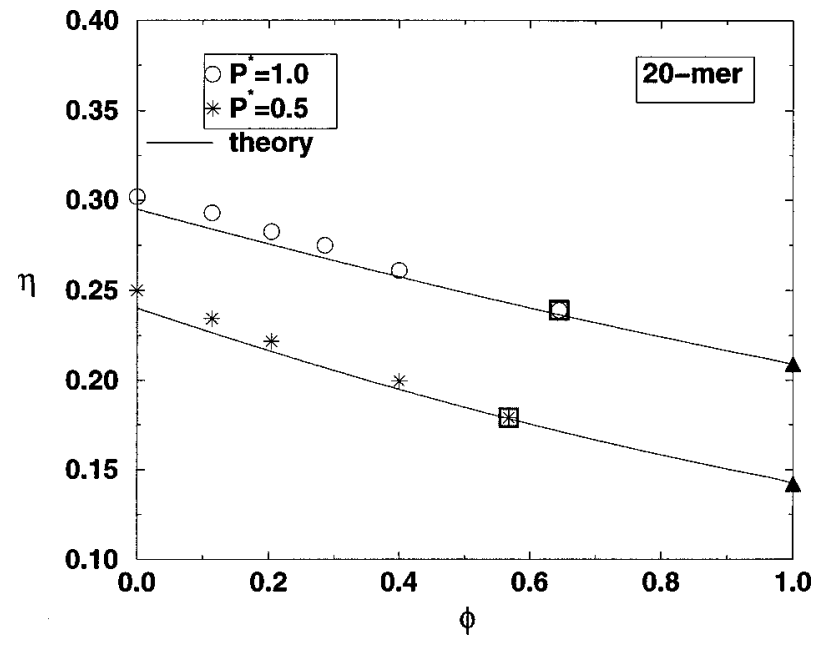

FIG. 4. Simulation results for the gel packing fraction, $\eta$, versus the solvent fraction, $\phi$, as the gel evolves towards swelling equilibrium. Data are shown for $P^{*}=0.5$ and $P^{*}=1.0$. The data points marked by squares represent the equilibrium swelling solvent and packing fractions, and the data points marked by solid triangles represent the pure solvent packing fraction [evaluated from the Carnahan-Starling equation of state (Ref. 23)]. The lines represent our theoretical predictions.

fraction, $\eta$, versus the solvent fraction, $\phi$, at $P^{*}=0.5$ and $P^{*}=1.0$, as obtained from Table VII. The data points represented by squares are the equilibrium swelling values for the packing fraction and solvent fraction at that bulk pressure, and the data points represented by the filled triangles (at $\phi=1.0$ ) are the pure solvent packing fractions (obtained using the Carnahan-Starling equation of state). ${ }^{23}$ The gel packing fraction decreases continuously as the solvent fraction increases, i.e., as the gel absorbs solvent [due to the chemical potential gradient), its volume increases in order to maintain the gel pressure constant, thus resulting in a decrease in packing fraction. For all values of $\phi$ at a given pressure, the value of $\eta$ lies between the dry network packing fraction (at $\phi=0$ ) and the bulk solvent packing fraction (at $\phi=1.0)]$. The lines in the figure represent our theoretical predictions which show good overall agreement with the simulation data.

Finally we examine the structural characteristics of the network chains in an effort to ascertain the validity of the affine assumption. The affine assumption states that when the gel swells, the end-to-end vectors of the individual chains deform proportionally with macroscopic deformation, i.e., $\left\langle R^{2}\right\rangle \propto \lambda^{2}$, where $\left\langle R^{2}\right\rangle$ is the mean-squared end-to-end distance of the network chains. This assumption was first employed in the affine network model of Flory and Wall, and in the phantom model of James and Guth, where it was used to describe the deformation of "phantom" network chains, i.e., chains for which the excluded-volume condition is neglected. Due to its simplicity, most subsequent theories of rubber elasticity, including ours, have used the affine assumption. The validity of the affine assumption is examined in Fig. 5, which displays the scaled mean-squared end-to-end distance of the network chains, $\left\langle R^{2}\right\rangle / \sigma^{2}$ as calculated in Table VII versus $\lambda^{2}$, on a logarithmic scale (which is useful for deducing the scaling exponents). The dashed line represents the affine assumption prediction and the solid lines are 


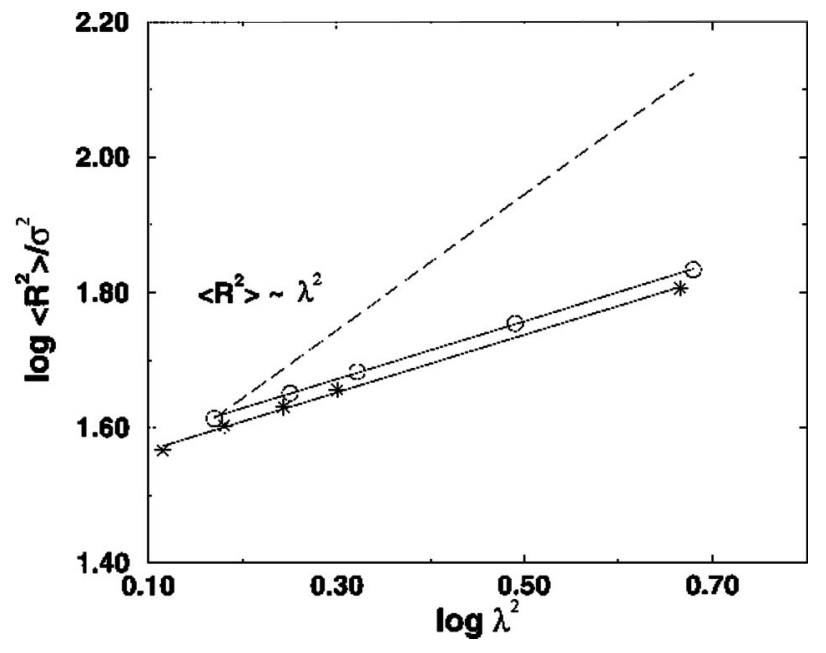

FIG. 5. Simulation results for the mean-squared end-to-end distance of the network chains, $\left\langle R^{2}\right\rangle$ versus the squared macroscopic deformation ratio, $\lambda^{2}$, for the 20-mer network chains, at $P^{*}=0.5$ (stars) and $P^{*}=1.0$ (circles). The solid lines represent linear regression fits to the data, and the dashed line represents the affine model prediction.

linear regression fits through the simulation data. It appears that the affine scaling is not followed either at $P^{*}=0.5$ or at $P^{*}=1.0$. In fact, the data at both pressures appears to follow the considerably weaker scaling of $\left\langle R^{2}\right\rangle \propto \lambda^{2 g}$, where $g$ is considerably less than 1 . The value of $g$ is 0.643 for the 20-mer network and 0.639 for the 35 -mer network. There could be several reasons for the failure of the affine assumption in these real networks: the constraints imposed by the excluded volume of the network chains on the chain deformation, the presence of trapped or temporary entanglements (this is relatively unlikely since we are working with low molecular weight systems), and the damping effect of solvent molecules.

\section{CONCLUSION}

In this paper, a combined DMD-MC technique has been used to investigate the swelling of polymer gels. A simple, analytical, mean-field theory has been proposed to predict the swelling properties of athermal gels, based on treating the free energy of the gel as the sum of the free energy of a reference polymer-solvent mixture and an elastic free energy contribution.

Our theory performs extremely well in predicting the gel packing fraction at swelling equilibrium, and fairly well in predicting the solvent fraction. The success of the theory is remarkable considering the simplicity of our elastic free energy term, which is basically an affinelike term that does not account for excluded volume, entanglements, etc. We attribute the success of our theory largely to an appropriate choice of the reference-polymer system that provides accurate predictions for the liquidlike contribution to the gel properties.

The DMD-MC technique is a simple, general, and fairly efficient method for investigating gel swelling. The coupling of the DMD technique with the gel swelling MC algorithm obviates the necessity for complex, network-specific MC moves that would otherwise be required in a purely $\mathrm{MC}$ network simulation. The efficiency of the combined technique is an important point in its favor, since the swelling process requires extremely large equilibration periods. However, despite the efficiency of our code, we found it difficult to simulate the swelling of long chain length networks (chain lengths of 50 or more). Part of the difficulty lies in the fact that the system size grows rapidly with the swelling of the gel; for instance, a 35-mer dry network of 90 chains (3150 segments) absorbs more than 9000 solvent particles during swelling at a pressure, $P^{*}=3.0$, resulting in a fairly large system of about 12000 segments.

The primary significance of this work lies in the fact that it shows that a simple theoretical approach can capture the main features of gel swelling. In addition, it provides previously unavailable simulation data for the swelling of trifunctional, continuous-space gels. We consider this study to be a starting point for detailed investigations of gel swelling, with possible future studies including the investigation of the swelling of networks with temperature dependent interaction potentials, the study of gel swelling in solvent mixtures, the examination of the effects of solvent size and structure, and of network structure (for example, the influence of network functionality, and structural irregularities) on the swelling properties of gels.

\section{ACKNOWLEDGMENTS}

The authors thank Dr. Paresh U. Kenkare for the use of his phase equilibria code and for many helpful discussions. This work was supported by the GAANN Computational Sciences Fellowship of the U.S. Department of Education, and the Office of Energy Research, U.S. Department of Energy under Contract No. DE-FG05-91ER14181. Acknowledgement is made to the Donors of the Petroleum Research Fund administered by the American Chemical Society. We thank the North Carolina Supercomputing Center for grants of CPU time on the Cray Y-MP and T90, and on the workstation cluster.

${ }^{1}$ R. Dagani, Chem. Eng. News 75, 26 (1997).

${ }^{2}$ N. R. Kenkare, C. K. Hall, and S. A. Khan, J. Chem. Phys. 110, 7556 (1999).

${ }^{3}$ P. J. Flory and J. Rehner, J. Chem. Phys. 11, 529 (1943).

${ }^{4}$ P. J. Flory, Principles of Polymer Chemistry (Cornell University Press, Ithaca, 1953).

${ }^{5}$ P. J. Flory and J. Rehner, J. Chem. Phys. 11, 512 (1943).

${ }^{6}$ F. T. Wall, J. Chem. Phys. 11, 527 (1943).

${ }^{7}$ M. Gottlieb, in Biological and Synthetic Polymer Networks, edited by O. Kramer (Elsevier Applied Science, London and New York, 1986).

${ }^{8}$ M. M. Prange, H. H. Hooper, and J. M. Prausnitz, AIChE J. 35, 803 (1989).

${ }^{9}$ M. Marchetti, S. Prager, and E. Cussler, Macromolecules 23, 1760 (1990).

${ }^{10}$ C. Panayiotou and I. Sanchez, Polymer 33, 5090 (1992).

${ }^{11}$ J. Gao and J. H. Weiner, Macromolecules 20, 2520 (1987).

${ }^{12}$ J. Gao and J. H. Weiner, Macromolecules 20, 2525 (1987).

${ }^{13}$ J. H. Weiner and J. Gao, in Molecular Basis of Polymer Networks, edited by A. Baumgartner and C. E. Picot (Springer-Verlag, New York, 1989).

${ }^{14}$ E. R. Duering, K. Kremer, and G. Grest, Phys. Rev. Lett. 67, 3531 (1991).

${ }^{15}$ E. R. Duering, K. Kremer, and G. S. Grest, Prog. Colloid Polym. Sci. 90, 13 (1992).

${ }^{16}$ G. S. Grest, K. Kremer, and E. R. Duering, Physica A 194, 330 (1993).

${ }^{17}$ E. R. Duering, K. Kremer, and G. Grest, J. Chem. Phys. 101, 8169 (1994).

${ }^{18}$ N. R. Kenkare, S. W. Smith, C. K. Hall, and S. A. Khan, Macromolecules 31, 5861 (1998). 
${ }^{19}$ H. L. Trautenberg, J. U. Sommer, and D. Goritz, J. Chem. Soc., Faraday Trans. 91, 2649 (1995).

${ }^{20}$ F. A. Escobedo and J. J. de Pablo, J. Chem. Phys. 104, 4788 (1996).

${ }^{21}$ F. A. Escobedo and J. J. de Pablo, J. Chem. Phys. 106, 793 (1997).

${ }^{22}$ F. A. Escobedo and J. J. de Pablo, Mol. Phys. 90, 437 (1997).

${ }^{23}$ N. F. Carnahan and K. E. Starling, J. Chem. Phys. 51, 635 (1969).

${ }^{24}$ F. A. Escobedo and J. J. de Pablo, J. Chem. Phys. 103, 3196 (1995).

${ }^{25}$ K. G. Honnell and C. K. Hall, J. Chem. Phys. 95, 4481 (1991).

${ }^{26}$ S. W. Smith, C. K. Hall, and B. D. Freeman, J. Chem. Phys. 104, 5616 (1996).

${ }^{27}$ S. W. Smith, C. K. Hall, and B. D. Freeman, J. Comput. Phys. 134, 16 (1997).

${ }^{28}$ D. C. Rapaport, J. Chem. Phys. 71, 3299 (1979).

${ }^{29}$ A. Bellemans, J. Orban, and D. V. Belle, Mol. Phys. 39, 781 (1980).

${ }^{30}$ B. J. Alder and T. E. Wainwright, in Symposium on Transport Processes In Statistical Mechanics, edited by I. Prigogine (Interscience, New York, 1956).

${ }^{31}$ B. J. Alder and T. E. Wainwright, J. Chem. Phys. 31, 459 (1959).

${ }^{32}$ J. M. Haile, Molecular Dynamics Simulation: Elementary Methods (Wiley, New York, 1992).
${ }^{33}$ J. Scanlan, J. Polym. Sci. 43, 501 (1960).

${ }^{34}$ D. S. Pearson and W. W. Graessley, Macromolecules 11, 528 (1977).

${ }^{35}$ M. R. Wilson and M. P. Allen, Mol. Phys. 80, 277 (1993).

${ }^{36}$ M. P. Allen and D. J. Tildesley, Computer Simulation of Liquids (Clarendon, Oxford, 1987).

${ }^{37}$ B. Widom, J. Chem. Phys. 39, 2808 (1963).

${ }^{38}$ D. Frenkel and B. Smit, Understanding Molecular Simulation: From Algorithms to Applications (Academic, San Diego, 1996).

${ }^{39}$ H. M. James and E. Guth, J. Chem. Phys. 11, 455 (1943).

${ }^{40}$ P. G. de Gennes, Scaling Concepts in Polymer Physics (Cornell University Press, Ithaca, NY, 1979).

${ }^{41}$ R. Dickman and C. K. Hall, J. Chem. Phys. 85, 4108 (1986).

${ }^{42}$ C. K. Hall, M. A. Denlinger, and K. G. Honnell, Fluid Phase Equilibria 53, 151 (1989).

${ }^{43}$ K. G. Honnell and C. K. Hall, J. Chem. Phys. 90, 1841 (1989).

${ }^{44}$ J. Alejandre and G. A. Chapela, Mol. Phys. 61, 1119 (1987).

${ }^{45}$ A. Yethiraj and C. K. Hall, J. Chem. Phys. 94, 3943 (1991).

${ }^{46}$ D. J. Tildesley and W. B. Streett, Mol. Phys. 41, 85 (1980).

${ }^{47}$ P. U. Kenkare, C. K. Hall, and C. Caccamo, J. Chem. Phys. 103, 8098 (1995) 